\title{
Role of metallothionein in murine experimental colitis
}

\author{
TOSHIFUMI TSUJI $^{1}$, YUJI NAITO ${ }^{1}$, TOMOHISA TAKAGI $^{1}$, MUNEHIRO KUGAI $^{1}$, HIROYUKI YORIKI $^{1}$, \\ RYUSUKE HORIE $^{1}$, AKIFUMI FUKUI ${ }^{1}$, KATSURA MIZUSHIMA ${ }^{1}$, YASUKO HIRAI ${ }^{1}$, KAZUHIRO KATADA ${ }^{1}$, \\ KAZUHIRO KAMADA ${ }^{1}$, KAZUHIKO UCHIYAMA ${ }^{1}$, OSAMU HANDA ${ }^{1}$, HIDEYUKI KONISHI ${ }^{1}$, \\ NOBUAKI YAGI ${ }^{1}$, HIROSHI ICHIKAWA ${ }^{1}$, RIE YANAGISAWA ${ }^{3}$, JUNKO S. SUZUKI ${ }^{4}$, \\ HIROHISA TAKANO ${ }^{5}$, MASAHIKO SATOH ${ }^{2}$ and TOSHIKAZU YOSHIKAWA ${ }^{1}$ \\ ${ }^{1}$ Department of Molecular Gastroenterology and Hepatology, Kyoto Prefectural University of Medicine, Kyoto 602-8566; \\ ${ }^{2}$ Laboratory of Pharmaceutical Health Sciences, School of Pharmacy, Aichi Gakuin University, Nagoya 464-8650; \\ ${ }^{3}$ Center for Environmental Health Sciences, ${ }^{4}$ Center for Environmental Risk Research, \\ National Institute for Environmental Studies, Tsukuba 305-8506; ${ }^{5}$ Kyoto University, \\ Graduate School of Engineering, Kyoto 615-8530, Japan
}

Received November 15, 2012; Accepted January 7, 2013

DOI: 10.3892/ijmm.2013.1294

\begin{abstract}
Metallothioneins (MTs) are a family of cysteine-rich low molecular-weight proteins that can act as reactive oxygen species scavengers. Although it is known that the induction of MT expression suppresses various inflammatory disorders, the role of MTs in intestinal inflammation remains unclear. In this study, we investigated the effects of dextran sulfate sodium (DSS) administration in mice with targeted deletions of the MT-I/II genes. Acute colitis was induced by $2 \%$ DSS in male MT-I/II double knockout (MT-null) and C57BL/6 (wild-type) mice. The disease activity index (DAI) was determined on a daily basis for each animal, and consisted of a calculated score based on changes in body weight, stool consistency and intestinal bleeding. Histology, colon length, myeloperoxidase (MPO) activity and colonic mRNA expression and the concentration of inflammatory cytokines were evaluated by real-time-PCR and enzyme-linked immunosorbent assay (ELISA). The localization of MTs and macrophages was determined by immunohistological and immunofluorescence staining. To investigate the role of MTs in macrophages, peritoneal macrophages were isolated and their responses to lipopolysaccharide were measured. Following DSS administration, the DAI score increased in a time-dependent manner and was significantly enhanced in the MT-I/II knockout mice. Colonic MPO activity levels and inflammatory cytokines [tumor necrosis factor (TNF)- $\alpha$, interferon (IFN)- $\gamma$ and interleukin (IL)-17] production increased following DSS administration, and these increases were significantly
\end{abstract}

Correspondence to: Dr Yuji Naito, Department of Molecular Gastroenterology and Hepatology, Graduate School of Medical Science, Kyoto Prefectural University of Medicine, 465 Kajii-cho, Kawaramachi-Hirokoji, Kamigyo-ku, Kyoto 602-8566, Japan

E-mail: ynaito@koto.kpu-m.ac.jp

Key words: metallothioneins, dextran sulfate sodium, intestinal inflammation, macrophages enhanced in the MT-I/II knockout mice compared with the wild-type mice. MT-positive cells were detected in the lamina propria and submucosal layer by immunohistochemical and immunofluorescence staining, and were mainly co-localized in F4/80-positive macrophages. The production of inflammatory cytokines (TNF- $\alpha$, IFN- $\gamma$ and IL-17) from isolated peritoneal macrophages increased following lipopolysaccharide stimulation, and these increases were significantly enhanced in the macrophages obtained from the MT-I/II knockout mice. These data indicate that MTs play an important role in the prevention of colonic mucosal inflammation in a mouse model of DSS-induced colitis, thus suggesting that endogenous MTs play a protective role against intestinal inflammation.

\section{Introduction}

Inflammatory bowel diseases (IBDs) such as ulcerative colitis and Crohn's disease are known as refractory and recurrent diseases of the gastrointestinal tract. Although the features of IBDs suggest a number of possible causes, including genetic, infectious and immunological factors, the precise pathogenesis of IBDs remains unknown (1-3). Recent evidence suggests that oxidative stress caused by reactive oxygen species (ROS) is an important factor involved in the onset and development of intestinal inflammation. Furthermore, it has also been demonstrated that disruptions in the antioxidant defense system are involved in the pathophysiology of IBDs $(4,5)$. Therefore, it is important to investigate the oxidative stress-related pathogenesis of IBDs.

Metallothioneins (MT) are a family of low molecular weight proteins containing multiple cysteine residues that coordinate multiple zinc and copper atoms, enabling a high affinity for monovalent and divalent heavy metal atoms (6). The presence of MTs has been confirmed in mammals, birds, fish, amphibians, reptiles and invertebrates, as well as in a wide range of plants, and eukaryotic and prokaryotic microorganisms (7). In mice, 4 MT isoforms have been identified: MT-I and MT-II are ubiquitously expressed, MT-III is expressed predominantly in the brain and MT-IV is expressed in stratified squamous epithelial cells $(8,9)$. 
In humans, MTs are encoded by at least 10 identified functional genes, and the encoded proteins are conventionally subdivided into 4 groups: MT-I, MT-II, MT-III and MT-IV (10). As MTs have a high affinity for metals, as noted above, MTs have free radical-scavenging potential, with MT-I/II exerting particularly potent antioxidant effects in comparison with the other isoforms.

As regards the role of MTs in various pathophysiological conditions causing inflammation, MTs have been reported to play a protective role in various animal models, including lipopolysaccharide (LPS)-induced lung injury (11), rheumatoid arthritis (12), multiple sclerosis (13), coagulatory disturbance (14), ethanol-induced gastroduodenal mucosal injury (15) and Helicobacter pylori-induced gastritis (16). MT-deficient mice are more susceptible to LPS-induced lethal shock following D-galactosamine (D-GalN) sensitization through the reduction of $\alpha 1$-acid glycoprotein (17). By contrast, there are conflicting data in a tumor necrosis factor (TNF)induced lethal shock model suggesting a reduction in mortality in MT-deficient mice, as compared to wild-type mice (18). Furthermore, MT-overexpressing mice are also more sensitive to the lethal effects of TNF compared to wild-type mice (19).

Although MTs are known to a play protective role in intestinal inflammation, studies on experimental colitis models using MT-deficient mice have revealed that MTs do not protect against the development of colitis. Tran et al (20) demonstrated that MT-deficient mice had significantly lower disease activity index (DAI) than wild-type mice in a dextran sodium sulfate (DSS)-induced colitis model, a widely accepted model of human IBD. Oz et al (19) also reported that there were no differences in histological damage following treatment with DSS among wild-type, MT-deficient and MT transgenic mice. Hence, the function of MTs in intestinal inflammation remains uncertain.

In the present study, we examined the role of MTs in intestinal inflammation using a mouse model of DSS-induced colitis, which is recognized as a useful experimental model of intestinal inflammation and is considered to be similar to human IBD (21-24).

\section{Materials and methods}

Animals. We used 7-week-old male MT-I/II double knockout (MT null) mice (25) and age-matched C57BL/6 wild-type mice. MT-I/II knockout mice were kindly provided by Dr Hirohisa Takano (Center for Environmental Health Sciences, National Institute for Environmental Studies, and Kyoto University, Graduate School of Engineering, Kyoto, Japan). C57BL/6 mice were purchased from Shimizu Laboratory Supplies Co., Ltd. (Kyoto, Japan). MT-I/II knockout mice were routinely bred in the vivarium of Kyoto Prefectural University of Medicine. Mice were housed in cages under a 12-h light/dark cycle, and a controlled temperature of $22^{\circ} \mathrm{C}$ and negative atmospheric pressure. Mice were maintained in a specific pathogen-free environment and provided with tap water and food ad libitum. Mice were fed a rodent diet (CE-2; Nihon CLEA, Tokyo, Japan) during the experiment. All experimental procedures were carried out in accordance with the National Institutes of Health (NIH) guidelines for the Care and Use of Laboratory Animals. Experimental protocols were approved by the Animal Care and Use Committee of Kyoto Prefectural University of Medicine (Kyoto, Japan).
Induction of colitis by DSS. Male wild-type mice and MT-I/II knockout mice ( 7 weeks old) were randomized into control and experimental groups (6-8 mice in each group). Experimental acute colitis was induced by treatment with $2.0 \%$ DSS (molecular weight 1,000-9,000, Lot SDR4219; Wako Pure Chemical Industries, Osaka, Japan) in drinking water for 7 days, as reported previously $(26,27)$. Mice were sacrificed under anesthesia 7 days after DSS treatment, and the colons were removed for macroscopic and histological examination. Colonic specimens were also obtained for biochemical assay and RNA isolation.

Evaluation of the severity of colitis. DAI, colon length and histology were analyzed. DAI was determined by scoring changes in animal weight, occult blood positivity, gross bleeding and stool consistency, as described previously $(26,28,29)$. We used 5 grades of weight loss ( 0 , no loss or weight gain; 1, 1-5\% loss; 2, 5-10\% loss; 3, 10-20\% loss; 4, >20\% loss), 3 grades of stool consistency ( 0 , normal; 2 , loose; and 4 , diarrhea), and 3 grades of occult blood ( 0 , negative; 2 , occult blood-positive; and 4 , gross bleeding) based on previous studies $(26,27)$. After determining DAI, the mice were sacrificed, and the entire colon was removed from the cecum to the anus, and colon length was measured as an indirect marker of inflammation.

Immediately after dissection, the distal colon was fixed in $10 \%$ buffered formalin for histological analysis. Sections (4- $\mu \mathrm{m}$-thick) were prepared and stained with hematoxylin and eosin (H\&E). The slides were then examined and scored in a blinded manner using a previously published grading system $(30,31)$. Briefly, a combined score of inflammatory cell infiltration and tissue damage was determined as follows: as regards cell infiltration: score 0 , occasional inflammatory cells in the lamina propria; 1 , increased inflammatory infiltrate in the lamina propria predominantly at the base of crypts; 2 , confluence of inflammatory infiltrate extending into the mucosa; and 3 , transmural extension of inflammatory infiltrate. As regards tissue damage: score 0 , no mucosal damage; 1 , partial (up to $50 \%$ ) loss of crypts in large areas; 2 , partial to total 50-100\% loss of crypts in large areas, epithelium intact; and 3, total loss of crypts in large areas and epithelium lost. The total histological score represents the sum of the cell infiltration and tissue damage scores, and thus ranges from 0 to 6 .

Measurement of myeloperoxidase (MPO) activity. MPO activity in the colonic mucosa, an index of polymorphonuclear leukocyte accumulation, was determined using a modification of the method described by Grisham et al (32). A total of $2 \mathrm{ml}$ of mucosal homogenate was centrifuged at 20,000 x g for $15 \mathrm{~min}$ at $4^{\circ} \mathrm{C}$ to pellet the insoluble cellular debris. The pellet was then re-homogenized in an equivalent volume of $0.5 \%$ hexadecyltrimethylammonium bromide. Samples were centrifuged at $20,000 \mathrm{x} \mathrm{g}$ for $15 \mathrm{~min}$ at $4^{\circ} \mathrm{C}$, and the supernatants were saved. MPO activity was assessed by measuring the $\mathrm{H}_{2} \mathrm{O}_{2}$-dependent oxidation of 3,3',5,5'-tetramethylbenzidine. One unit of enzyme activity was defined as the amount of MPO that caused the absorbance to change by $1.0 / \mathrm{min}$ at $655 \mathrm{~nm}$ and $25^{\circ} \mathrm{C}$.

RNA analysis. The mRNA expression levels of MT-I/II, TNF- $\alpha$, interferon (IFN)- $\gamma$, interleukin (IL)-17 and $\beta$-actin (internal standard) were determined by real-time-PCR. Samples for mRNA isolation were removed from colonic tissue. Total RNA 
was isolated with the acid guanidinium phenol chloroform (AGPC) method using Isogen reagent (Nippon Gene, Toyama, Japan). RNA was stored at $70^{\circ} \mathrm{C}$ until it was used for reversetranscription polymerase chain reaction (RT-PCR). A total of $1 \mu \mathrm{g}$ of extracted RNA was reverse-transcribed into first-strand complementary DNA (cDNA) using the High Capacity cDNA Reverse Transcription kit (Applied Biosystems, Foster City, CA). Real-time PCR for CINC-1 and $\beta$-actin was carried out with the 7300 Real-Time PCR system (Applied Biosystems) using the DNA-binding dye SYBR ${ }^{\circledR}$-Green for the detection of PCR products. Primers had the following sequences: MT-I sense, 5'-GCTGTGCCTGATGTGACGAA-3' and antisense, 5'-AGGAAGACGCTGGGTTGGT-3'; MT-II sense, 5'-TGCGC TCGACCCAATACTC-3' and antisense, 5'-TCTAGGAGCG TGATGGAGAGAAG-3'; TNF- $\alpha$ sense, 5'-ATCCGCGACGT GGAACTG-3' and antisense, 5'-ACCGCCTGGAGTTCTG GAA-3'; IFN- $\gamma$ sense, 5'-CCTGCGGCCTAGCTCTGA-3' and antisense, 5'-CCATGAGGAAGAGCTGCAAAG-3'; IL-17 sense, 5'-TCATCTGTGTCTCTGATGCTGTTG-3' and antisense, 5'-TCGCTGCTGCCTTCACTGT-3'; and $\beta$-actin sense, 5'-GAGCAAACATCCCCCAAAGTT-3' and antisense, 5'-GCC GTGGATACTTGGAGTGACT-3'. Relative quantities of gene expression with real-time PCR data were calculated relative to $\beta$-actin.

Determination of colonic concentrations of TNF- $\alpha, I F N-\gamma$ and $I L-17$. We determined the concentrations of TNF- $\alpha$, IFN- $\gamma$ and IL-17 in the supernatant of colonic mucosal homogenates using an enzyme-linked immunosorbent assay (ELISA) kit for TNF- $\alpha$, IFN- $\gamma$ and IL-17 (R\&D Systems, Minneapolis, MN) according to the manufacturer's instructions. Subtractive readings at $550 \mathrm{~nm}$ from the readings at $450 \mathrm{~nm}$ were converted to $\mathrm{pg} / \mathrm{ml}$ using values obtained from standard curves generated with varying concentrations of recombinant TNF- $\alpha$, IFN- $\gamma$ and IL-17.

Immunohistochemical and immunofluorescence staining of MT and F4/80 in colonic mucosa. After 24-h fixation in formalin, samples were embedded in paraffin, after which $4-\mu \mathrm{m}$-thick sections were cut using a microtome cryostat and mounted on MAS-coated slides. We performed antigen retrieval using proteinase $\mathrm{K}$ solution, and the sections were rinsed with distilled water for $5 \mathrm{~min}$, followed by incubation with $3 \%$ hydrogen peroxide in methanol for 30 min to block endogenous peroxidase activity. After incubation, the sections were washed in phosphate-buffered saline (PBS)-Tween-20 for $5 \mathrm{~min}$. Non-specific binding was blocked by incubating the slides with Dako Cytomation protein block (Dako, Tokyo, Japan) for $30 \mathrm{~min}$ at room temperature. The sections were then incubated with primary antibody against MTs (Dako, Glostrup, Denmark) diluted at 1:50 and F4/80 (Novus Biologicals, Inc., Littleton, CO) diluted at 1:200 with antibody dilution (Dako) overnight at $4^{\circ} \mathrm{C}$. The sections were washed 3 times in PBS-Tween-20 for $5 \mathrm{~min}$ and incubated with secondary antibody [Histofine Simple Stain mouse MAX PO (rabbit); Nichirei Biosciences, Inc., Tokyo, Japan] for $30 \mathrm{~min}$ at room temperature. Unbound antibodies were removed by 3 washes in PBS for $5 \mathrm{~min}$, and bound antibodies were visualized using diaminobenzidine as a chromogen substrate reagent. Negative controls for non-specific binding incubated with secondary antibodies were confirmed to produce no signal. All sections were counterstained with hematoxylin. Sections were finally dehydrated, cleared and coverslipped.

For the immunofluorescence staining of MTs and F4/80 (diluted at 1:200; Novus Biologicals, Inc.), bound antibodies were visualized using the secondary antibodies, anti-rabbit Alexa 594 (Molecular Probes, Inc., Eugene, OR) and anti-rat Alexa 488 (Molecular Probes), respectively, diluted at 1:1,000. Negative controls used for non-specific binding and incubated with secondary antibodies were confirmed to produce no signal. Fluorescence staining was observed under an inverted fluorescence microscope (IX70-23FL/DIC-SP; Olympus, Tokyo, Japan).

F4/80-positive cells in colonic mucosa. We evaluated the number of F4/80-positive cells in the intestinal mucosa of wild-type and MT-I/II knockout mice following DSS-induced colitis. The number of positively stained cells in the intestinal mucosa was counted in 50 high-power fields per section under a microscope, as described previously (33).

Isolation of resident peritoneal macrophages and determination of cytokine production. Resident peritoneal macrophages were obtained by peritoneal lavage using $10 \mathrm{ml}$ of PBS from both wild-type and MT-I/II knockout mice. Cells were plated at $5 \times 10^{5}$ cells/well (24-well plate) and incubated for $2 \mathrm{~h}$ at $37^{\circ} \mathrm{C}$ in RPMI-1640 medium with 5\% fetal bovine serum (FBS), $2 \mathrm{mM}$ glutamine, $50 \mathrm{mg} / \mathrm{ml}$ streptomycin and $50 \mathrm{U} / \mathrm{ml}$ penicillin (all obtained from Life Technologies Corp.). The cells were washed twice with PBS to remove non-adherent cells and cultured in RPMI-1640 for $24 \mathrm{~h}$ at $37^{\circ} \mathrm{C}$. Subsequently, the cells were stimulated with LPS (Escherichia coli 055:B5; Sigma-Aldrich Corp., St. Louis, MO) at $10 \mathrm{ng} / \mathrm{ml}$. After a 12-h incubation with LPS, cell supernatants were collected and stored at $-70^{\circ} \mathrm{C}$. TNF- $\alpha$, IFN- $\gamma$ and IL-17 levels in culture supernatants were determined using an ELISA kit according to the manufacturer's instructions (R\&D Systems).

Statistical analysis. The results in this study are presented as the means \pm standard error of the mean (SEM). Overall differences between the groups were determined by one-way analysis of variance (ANOVA). If the results from one-way ANOVA were significant, the differences between individual groups were analyzed by Bonferroni's multiple comparisons test. P-values $<0.05$ were considered to indicate statistically significant differences. All analyses were performed using GraphPad Prism 5 software (GraphPad Software, San Diego, CA) on a Windows-based computer.

\section{Results}

Expression of $M T$ in inflamed colonic mucosa. In order to investigate MT-I/II expression in the inflamed mucosa following the administration of DSS, we examined the expression of colonic MT-I/II mRNA using real-time PCR analysis. The results confirmed that the expression of MT-I/II was significantly higher in the inflamed colonic mucosa in the model of DSS-induced colitis (Fig. 1A).

Body weight changes, DAI scores and colon length following DSS administration. The intake of DSS solution was moni- 


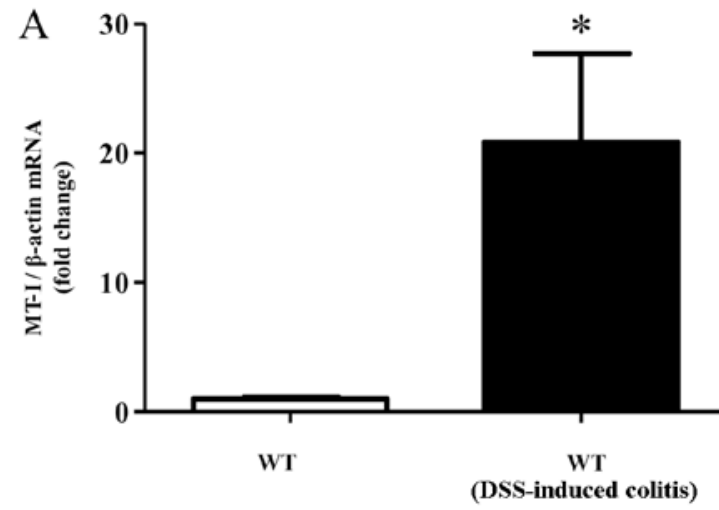

B

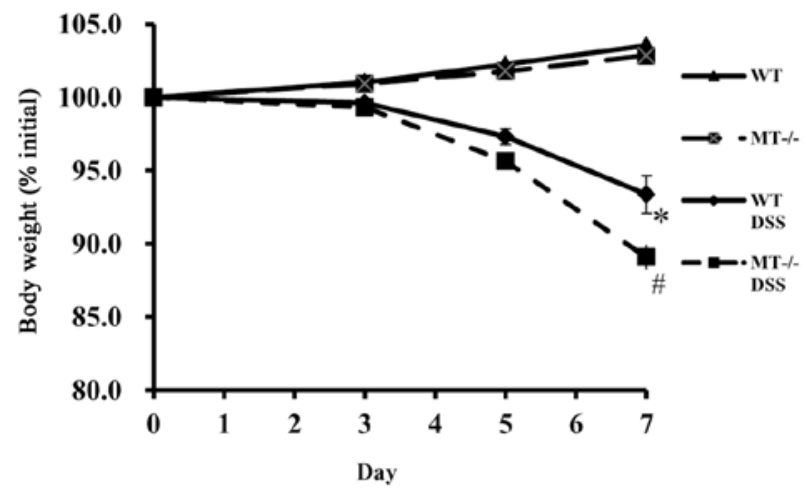

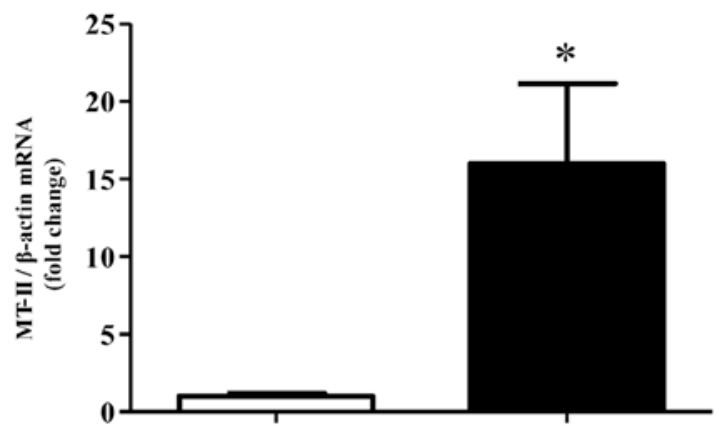

WT

(DSS-induced colitis)

C

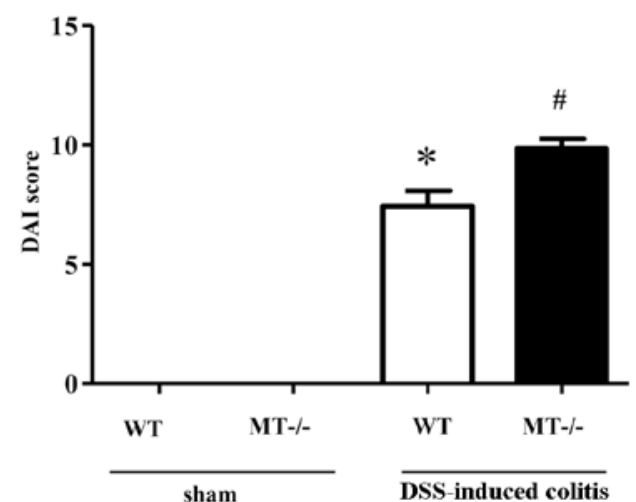

$\mathrm{D}$

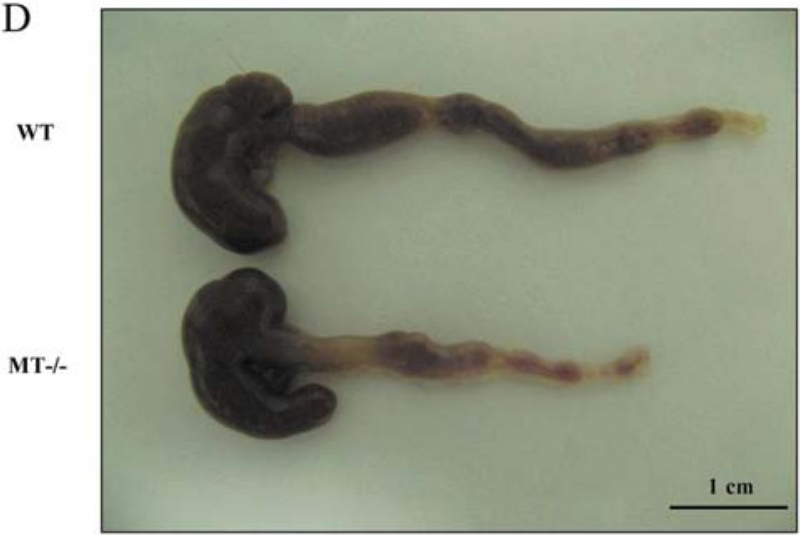

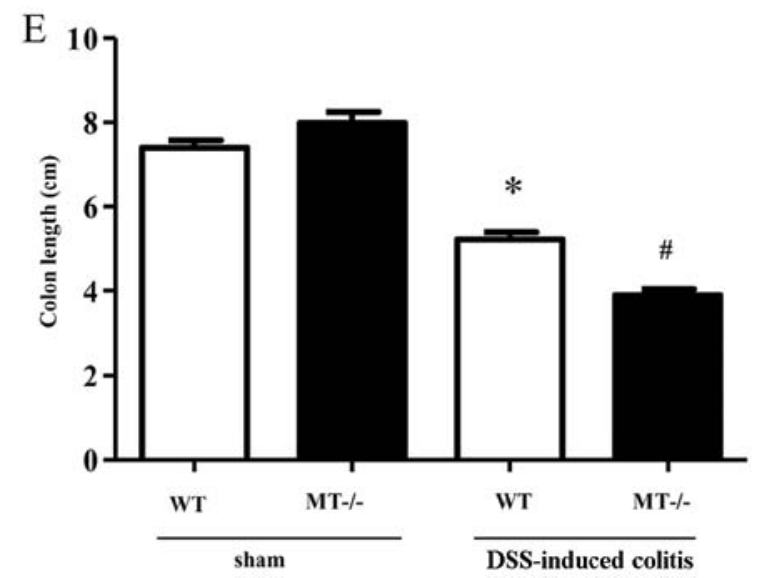

Figure 1. (A) Expression of colonic MT-I/II mRNA in normal mice and in the mouse model of dextran sulfate sodium (DSS)-induced colitis. Real-time PCR analysis confirmed the upregulation of MT-I/II expression following DSS-induced colitis. Both columns represent the means \pm standard error of the mean (SEM). $\mathrm{n}=8$. . $\mathrm{P}<0.05$ vs. wild-type mice. (B) Changes in body weight following treatment with DSS. MT-I/II knockout mice showed a marked decrease in body weight. ${ }^{*} \mathrm{P}<0.05$ vs. wild-type mice treated with the vehicle, ${ }^{*} \mathrm{P}<0.05$ vs. wild-type mice treated with DSS. (C) Disease activity index (DAI) of MT-I/II knockout mice was significantly higher than that of wild-type mice. Each column represents the means \pm SEM. $n=8$. " $\mathrm{P}<0.05$ vs. wild-type mice treated with the vehicle; ${ }^{\text {"}} \mathrm{P}<0.05$ vs. wild-type mice treated with DSS. (D) Representative macroscopic findings. MT-I/II knockout mice showed marked colon shortening. (E) Colon length following treatment with DSS. Shown is the average colon length. Colon length in MT-I/II knockout mice was significantly shorter than that of wild-type mice. Both columns represent the means \pm SEM. $n=8$. "P $<0.05$ vs. wild-type mice treated with the vehicle; ${ }^{\prime \prime} \mathrm{P}<0.05$ vs. wild-type mice treated with DSS. WT, wild-type; $\mathrm{MT}^{-}$, MT-I/II knockout (MT null) mice.

tored throughout the experiments and was found to be similar among the experimental groups (data not shown). The mice exposed to $2.0 \%$ DSS developed symptoms of acute colitis, with diarrhea accompanied by rectal bleeding and body weight loss. Decreases in body weight were significantly greater in the MT-I/II knockout mice in comparison to the wild-type mice (Fig. 1B). DAI scores in the DSS-treated mice, determined by weight loss, stool consistency and blood in stool, were significantly increased in the MT-I/II knockout mice compared to the wild-type mice (Fig. 1C).

Moreover, colon length is known to be reduced in DSS colitis along with the exacerbation of intestinal inflammation. In this study, MT-I/II knockout mice showed marked colon shortening 7 days after DSS administration (Fig. 1D). 
A
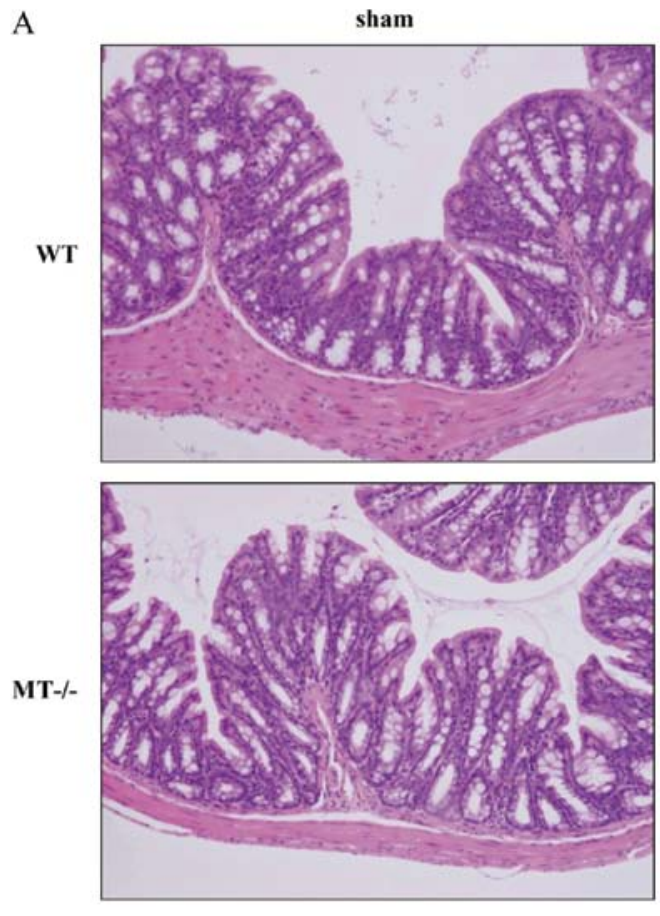
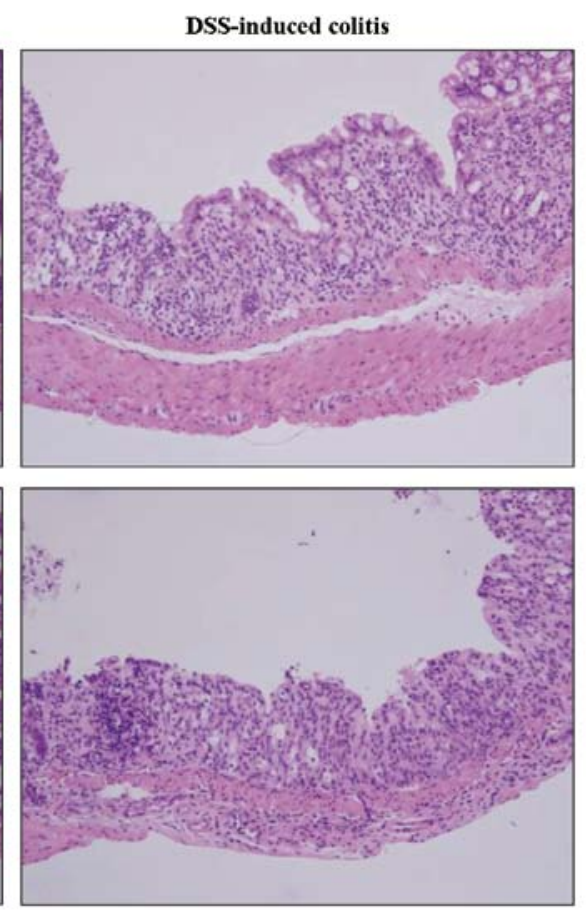
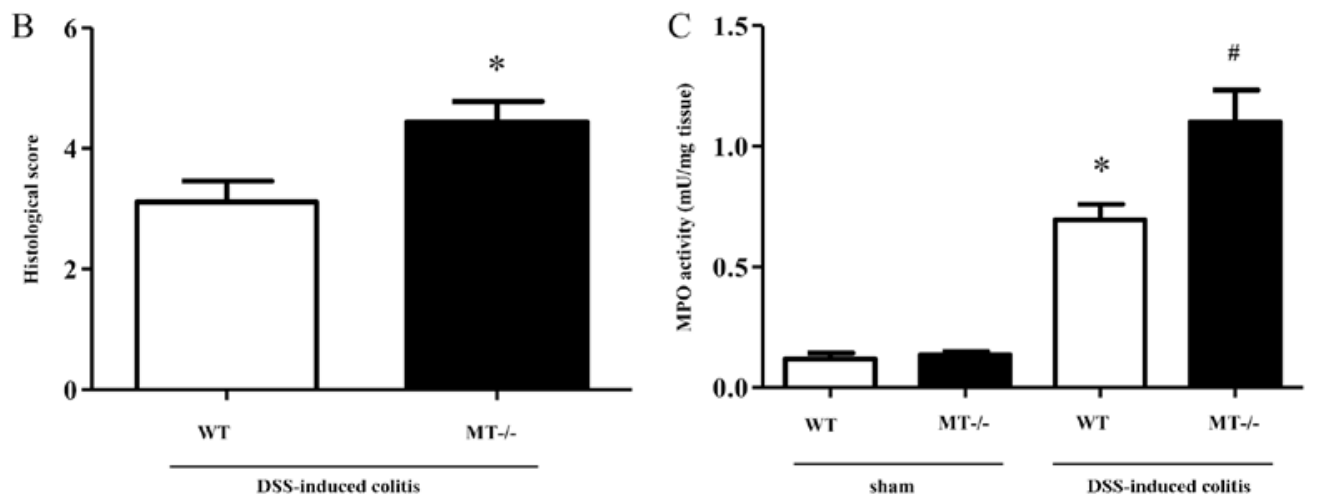

Figure 2. (A) Histological appearance of the colon in dextran sulfate sodium (DSS)-treated MT-I/II knockout mice. Magnification, $x 100$. Hematoxylin and eosin staining. (B) Histological scores of wild-type mice were significantly lower than those of MT-I/II knockout mice. (C) Levels of myeloperoxidase (MPO) activity were elevated in MT-I/II knockout mice, as compared to wild-type mice treated with DSS. Each column represents the means \pm SEM. $n=8$. ${ }^{*}<<0.05$ vs. wild-type mice treated with the vehicle; "P<0.05 vs. wild-type mice treated with DSS. WT, wild-type; MT-/-, MT-I/II knockout (MT null) mice.

Colon length following DSS administration was significantly decreased in the MT-I/II knockout mice compared to the wildtype mice (Fig. 1E). These data suggest that MTs play a crucial role in the development of intestinal inflammation.

Histology and neutrophil accumulation following DSS administration. The protective effects of MTs were also confirmed by histological analysis. Fig. 2A shows the typical histological appearance of wild-type and MT-I/II knockout mice. In the wild-type mice, the administration of $2 \%$ DSS for 7 days resulted in colonic ulceration associated with large areas of epithelial crypt loss and inflammatory cell infiltration throughout the mucosa. On the other hand, MT deficiency resulted in larger erosions with a high degree of inflammatory cell infiltration. The histological scores reflected these findings (Fig. 2B).

Tissue-associated MPO activity (as an index of polymorphonuclear leukocyte accumulation) in the colonic mucosa increased from $0.130 \pm 0.079 \mathrm{mU} / \mathrm{mg}$ protein (basal concentra- tion) to $0.903 \pm 0.114 \mathrm{mU} / \mathrm{mg}$ protein in the wild-type mice 7 days following the administration of DSS (Fig. 2B). MPO activity increased in the wild-type mice following DSS administration, and was significantly elevated in the DSS-treated MT-I/II knockout mice (Fig. 2B).

Colonic mRNA expression and mucosal concentrations of various inflammatory cytokines following DSS administration. The expression of inflammatory cytokines in the colonic mucosa following DSS treatment was determined using real-time PCR and ELISA. mRNA expression levels of inflammatory cytokines (TNF- $\alpha$, IFN- $\gamma$ and IL-17) were significantly increased in the colonic mucosal tissue of the MT-I/II knockout mice compared with the wild-type mice following the administration of DSS (Fig. 3). In agreement with these results, the colonic mucosal concentrations of these inflammatory cytokines (TNF- $\alpha$, IFN- $\gamma$ and IL-17) in the DSS-treated MT-I/II knockout mice were significantly higher, as compared with the DSS-treated wild-type mice (Fig. 4). 

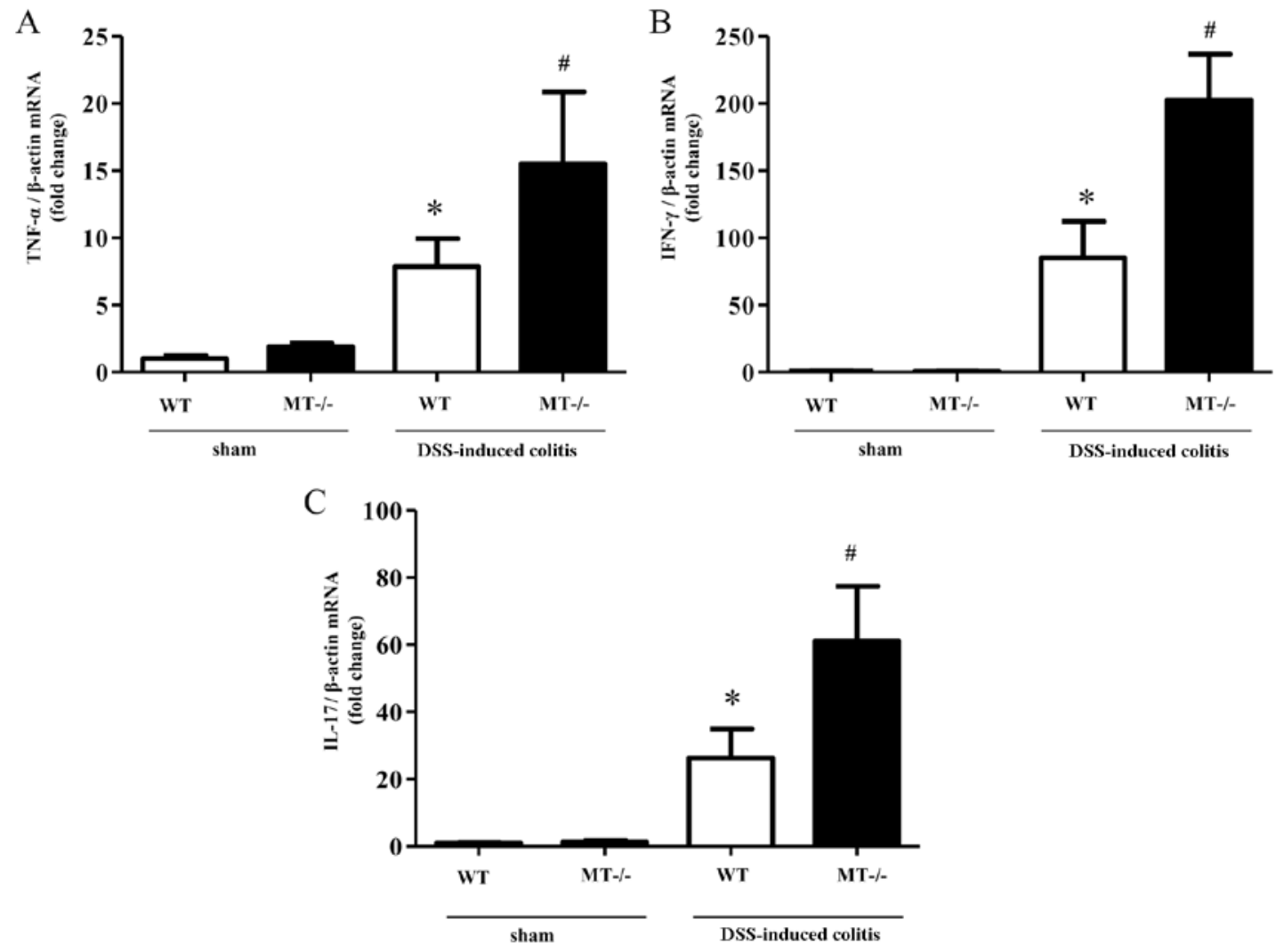

Figure 3. mRNA cytokine expression in colonic mucosal tissue. Inflammatory cytokine [(A) TNF- $\alpha$, (B) IFN- $\gamma$ and (C) IL-17] levels were significantly higher in colonic mucosal tissue of MT-I/II knockout mice. Each column represents the means \pm SEM. $n=8$. ${ }^{*}<<0.05$ vs. wild-type mice treated with the vehicle; ${ }^{\#} \mathrm{P}<0.05$ vs. wild-type mice treated with dextran sulfate sodium (DSS). WT, wild-type; MT-/-, MT-I/II knockout (MT null) mice.
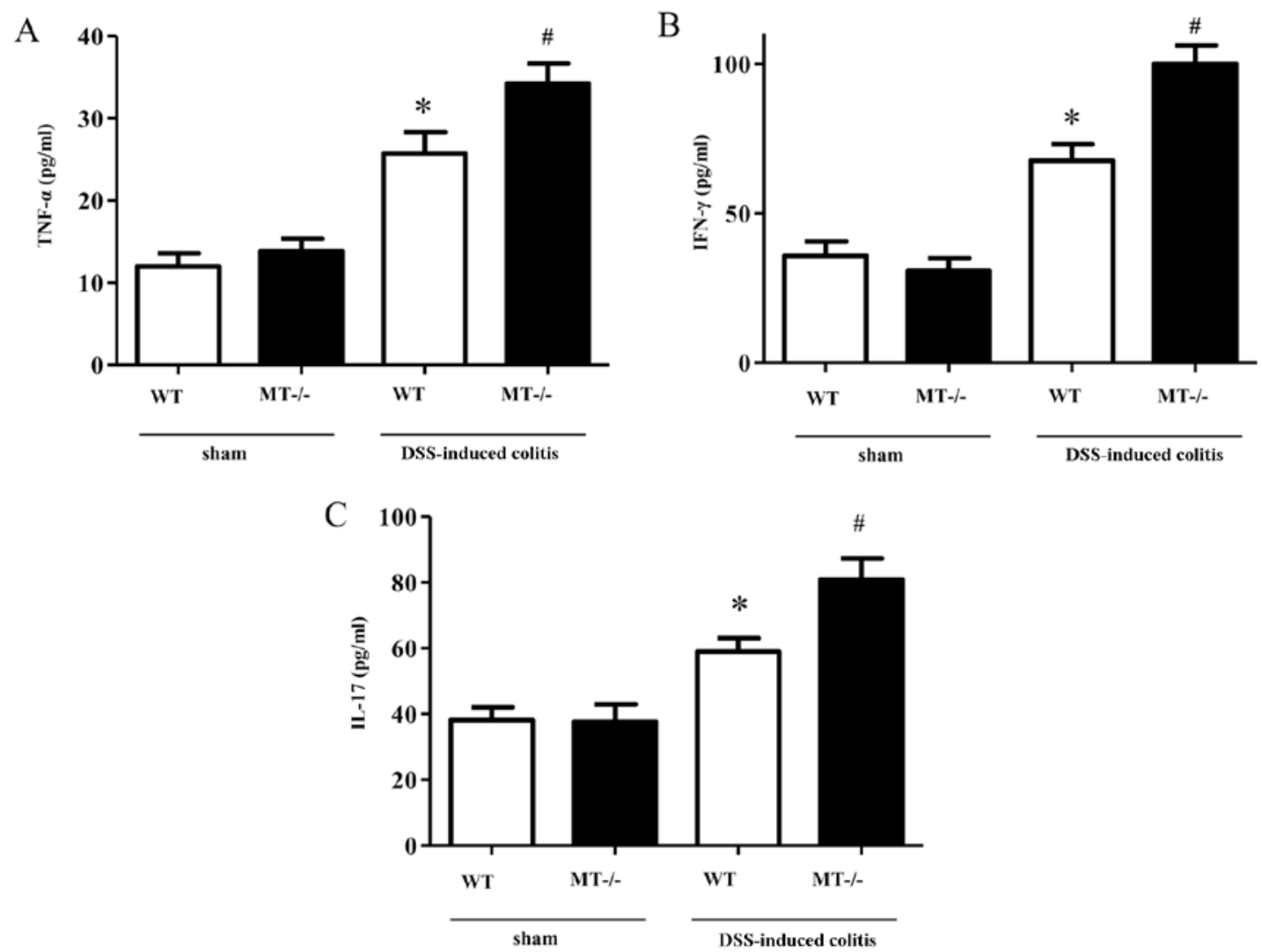

Figure 4. Production of cytokines in colonic mucosal tissue as shown by ELISA. Levels of inflammatory cytokines [(A) TNF- $\alpha$, (B) IFN- $\gamma$ and (C) IL-17] were significantly elevated in colonic mucosal tissue of MT-I/II knockout mice. Each column represents the means $\pm \mathrm{SEM}$. $\mathrm{n}=8$. ${ }^{*} \mathrm{P}<0.05$ vs. wild-type mice treated with the vehicle; ${ }^{~} \mathrm{P}<0.05$ vs. wild-type mice treated with dextran sulfate sodium (DSS). WT, wild-type; MT-/-, MT-I/II knockout (MT null) mice. 
A
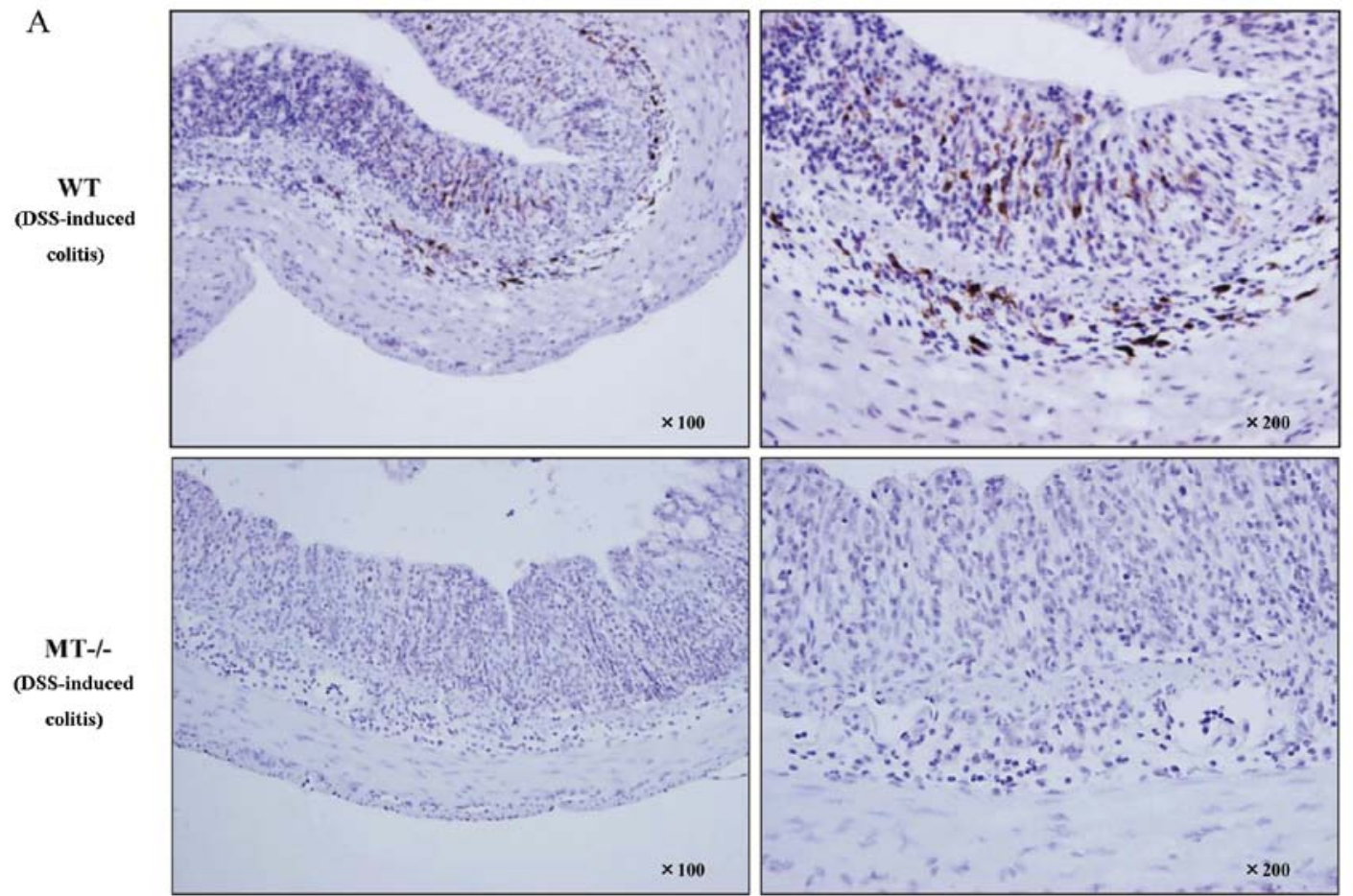

B

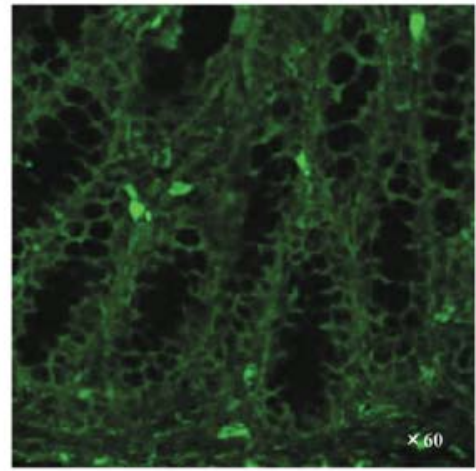

$\mathbf{F 4 / 8 0}$

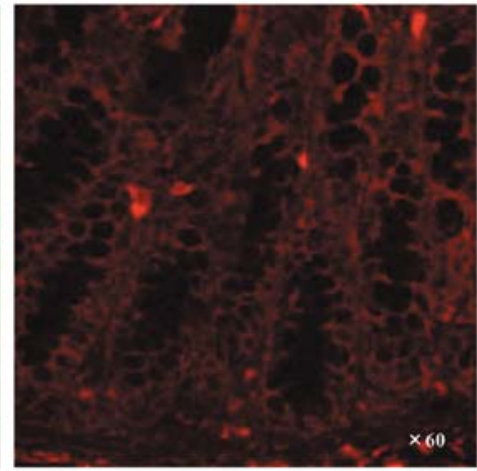

MT

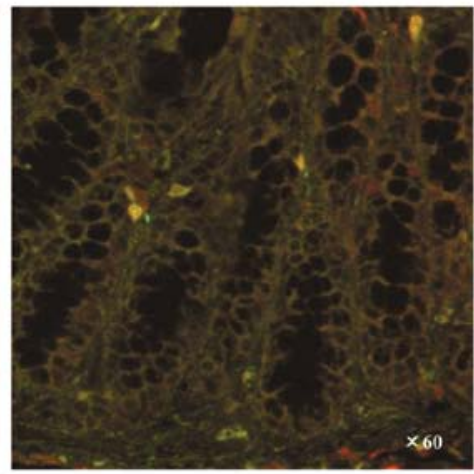

Merge

Figure 5. (A) Immunohistochemical staining of MTs. MT-positive cells were mainly localized in the lamina propria and submucosal layer as shown by immunohistochemical examination of wild-type mice, but MTs were not expressed in the MT-I/II knockout mice. (B) Localization of colonic MT expression was analyzed using immunofluorescence staining. MT and F4/80 were visualized under a laser scanning confocal microscope using the secondary antibodies, antirabbit Alexa 594 and anti-rat Alexa 488. A representative image of 3 separate experiments is shown. MT-positive cells mostly corresponded with F4/80-positive macrophages. WT, wild-type; MT-/-, MT-I/II knockout (MT null) mice.

Immunohistochemical and immunofluorescence staining of MTs in inflamed colonic mucosa. MTs are mainly localized in mononuclear cells in the lamina propria and submucosal layer, and these MT-positive cells were elevated in the DSS-treated wild-type mice. By contrast,MT-positive cells were not detected in the colons of MT-I/II knockout mice before and after DSS administration (Fig. 5A).

In addition, in order to investigate the detailed localization of MT expression in the colonic mucosa, we conducted immunofluorescence staining of the colonic mucosa. MT-positive cells largely corresponded with F4/80-positive macrophages (Fig. 5B).

Number of F4/80-positive cells in inflamed colonic mucosa. F4/80-positive cells were mainly localized in the lamina propria and submucosal layer in wild-type and MT-I/II knockout mice after DSS administration (Fig. 6A). Furthermore, we evaluated the number of the F4/80-positive cells in the intestinal mucosa of wild-type mice and MT-I/II knockout mice. No significant differences were observed in the number of F4/80positive cells between the DSS-treated wild-type and MT-I/II knockout mice (Fig. 6B).

Production of inflammatory cytokines in peritoneal macrophages following LPS treatment. In order to determine the production of inflammatory cytokines (TNF- $\alpha$, IFN- $\gamma$ and IL-17) in murine peritoneal macrophages following treatment with LPS, we measured the levels of these cytokines in the supernatant of cultured cells using ELISA. The concentrations of the inflammatory cytokines (TNF- $\alpha$, IFN- $\gamma$ and IL-17) 


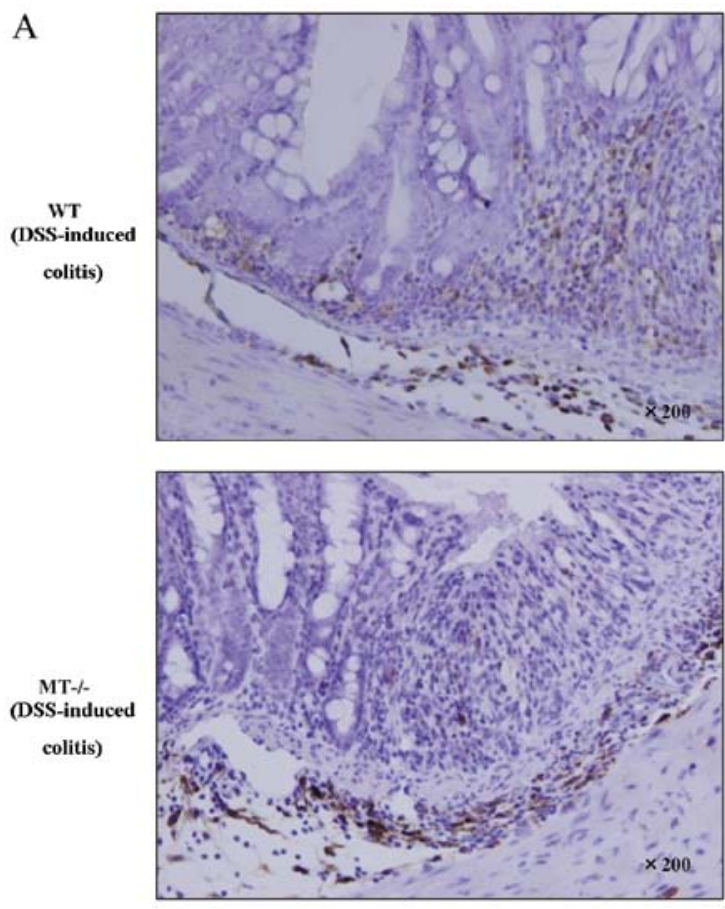

B

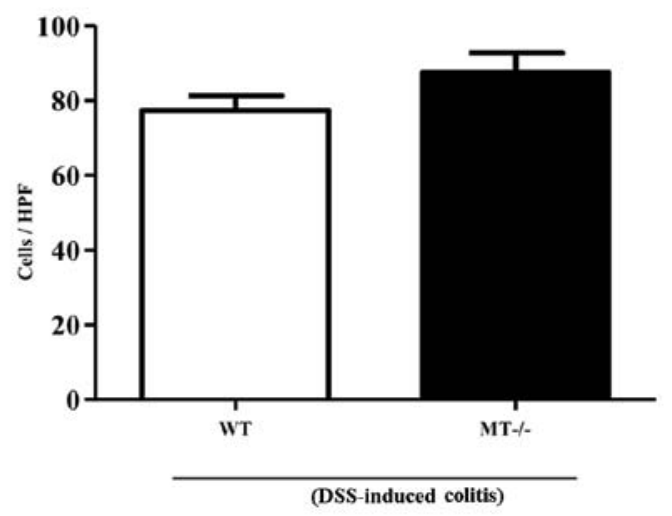

Figure 6. (A) Immunohistochemical staining of F4/80-positive macrophages. F4/80-positive cells were mainly localized in the lamina propria and submucosal layer as shown by immunohistochemical examination of wild-type and MT-I/II knockout mice. (B) The number of macrophages in wild-type and MT-I/II knockout mice was approximately equal. Cells/HPF, number of cells per high power field; WT, wild-type; MT-/-, MT-I/II knockout (MT null) mice.
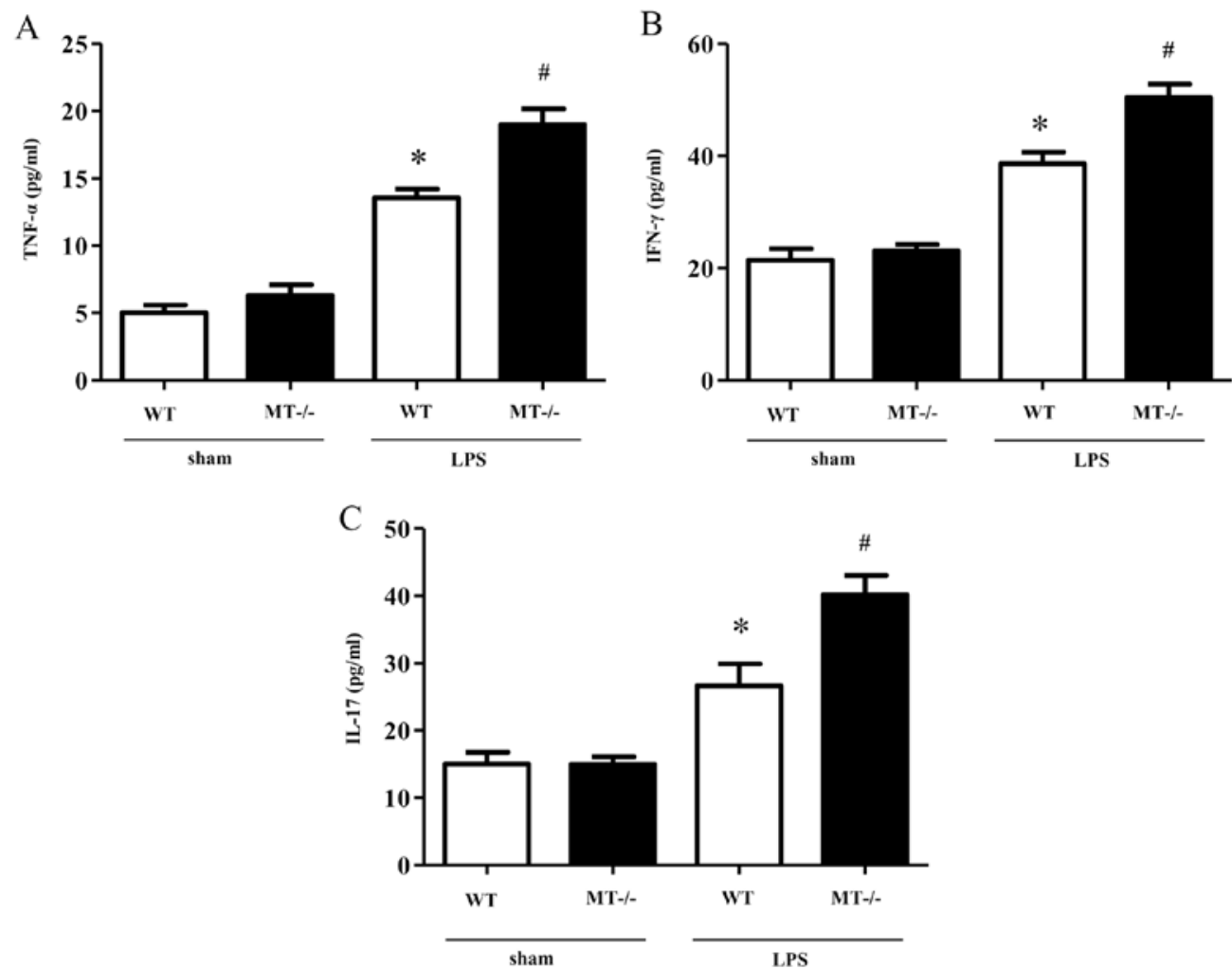

Figure 7. Production of cytokines in the supernatant of isolated peritoneal macrophages. Levels of inflammatory cytokines [(A) TNF- $\alpha$, (B) IFN- $\gamma$ and (C) IL-17] were significantly higher in the supernatants of macrophages derived from MT-I/II knockout mice when compared to those of wild-type mice. Each column represents the means \pm SEM. $n=8$. ${ }^{*} \mathrm{P}<0.05$ vs. wild-type mice treated with vehicle; ${ }^{*} \mathrm{P}<0.05$ vs. wild-type mice treated with lipopolysaccharide (LPS). WT, wild-type; MT-/-, MT-I/II knockout (MT null) mice.

in the LPS-treated macrophages isolated from the MT-I/II knockout mice were significantly higher as compared with the LPS-treated macrophages derived from the wild-type mice (Fig. 7). 


\section{Discussion}

In the current study, intestinal injury was assessed based on a variety of factors, including body weight, disease activity score, colon length and histology. In each assessment, colonic injury was found to be significantly aggravated in the DSS-treated MT-I/II knockout mice, as compared with the DSS-treated wild-type mice. Histological analysis also showed an enhanced infiltration of inflammatory cells, particularly neutrophils and mononuclear cells, as well as mucosal epithelial cell disruption in the DSS-treated MT-I/II knockout mice. Enhanced intestinal injury and neutrophil accumulation in MT-I/II knockout mice were also confirmed by the measurement of histological scores using a previously reported grading system $(30,31)$ and MPO activity of the colon, respectively.

A number of previous studies have described the role of MTs in the inflammatory response to DSS. Tran et al (20) first demonstrated that MT-deficient mice had significantly lower DAI when compared with wild-type mice in a model of DSS-induced colitis. However, the results from our study are not in agreement with the histological assessment made in the study by Tran et al. Another study by $\mathrm{Oz}$ et al (19) reported no differences in histological damage following treatment with DSS among wild-type mice, MT-deficient mice and MT transgenic mice. However, their study did not include the assessment of DAI. On the other hand, our data suggest that MT deficiency results in disease exacerbation in a mouse model of DSS-induced colitis, and that MTs play a protective role in the development of intestinal inflammation. While considering these results, we noted differences in the manufacture of DSS and vivarium in these studies, and it has been reported that these factors can lead to differences in the severity of DSS-induced colitis $(34,35)$.

As regards MT expression in intestinal inflammation, there are conflicting reports on the role of MTs in intestinal inflammation. Mulder et al (36) demonstrated that MT expression was decreased in the colonic mucosa of patients with IBD. Kruidenier et al (37) also reported that patients with IBD had decreased MT expression levels in the colon. By contrast, Lih-Brody et al (38) and Bruwer et al (39) reported that MT expression was markedly elevated in the colonic mucosa of patients with IBD. Similarly, MT-I/II expression was significantly higher in the inflamed colonic mucosa in mice with DSS-induced colitis, as compared with normal colonic mucosa in wild-type mice in the present study.

In the present study, in order to investigate the localization of MTs in the intestine, immunohistochemical staining was performed with murine colonic samples using antiMT-I/II antibody. MT-I/II expression was mainly observed in mononuclear cells in the lamina propria and submucosal layer of the murine intestine. Further examination using immunofluorescence staining revealed that these mononuclear cells were F4/80-positive macrophages. Although the localization of MTs in the gastrointestinal tract is not yet fully understood, Kruidenier et al (37) demonstrated that MTs are mainly expressed in the small intestinal epithelium, not in cells in the lamina propria. By contrast, a previous study by Al-Gindan et al (40) demonstrated that MT expression is localized in submucosal cells in the colonic mucosa in rats, similar to our results.
As regards the expression of MTs in macrophages, there is an extensive amount of evidence suggesting that alveolar macrophages show strong MT expression. Mehta et al (41) demonstrated that the reduction in MT expression induced by zinc deficiency in alveolar macrophages inhibits phagocytic function, indicating that MTs mediate innate immune function. Pankhurst et al (42) also reported that MT-expressing macrophages in the murine brain may play a crucial role in the regulation of $\mathrm{Th} 1 / \mathrm{Th} 2$ cytokine balance. In this study, the production of Th1, Th2 and Th17 cytokines was significantly elevated in the MT-I/II knockout mice, indicating that MTs possibly regulate the production of these cytokines. It is known that TNF- $\alpha$, IFN- $\gamma$ and IL-17 are mainly released by helper T cells (Th-1, Th-2 and Th-17) and macrophages $(43,44)$, and large amounts of cytokine expression lead to severe mucosal inflammation in ulcerative colitis and Crohn's disease (45). In the present study, we evaluated the function of macrophages derived from MT-deficient mice, as the number of infiltrating F4/80-positive macrophages in the intestinal mucosa of mice treated with DSS did not differ between the wild-type and MT-I/II knockout mice. We found that the production of inflammatory cytokines (TNF- $\alpha$, IFN- $\gamma$ and IL-17) in the supernatant of macrophages isolated from MT-I/II knockout mice following LPS stimulation was significantly higher compared to wild-type mice. Thus, MTs appear to play an important role in the anti-inflammatory function of macrophages and MT-expressing macrophages may play a protective role in the pathogenesis of IBDs.

In conclusion, our results suggest that MTs play a protective role against colonic mucosal inflammation in a mouse model of DSS-induced colitis through their anti-inflammatory function in macrophages, indicating that endogenous MTs play an important role in the protection of the intestinal mucosa. In the future, MTs may prove to be novel therapeutic target molecules in IBDs.

\section{Acknowledgements}

The authors thank Dr Chiharu Tohyama (The University of Tokyo) for providing the MT-I/II knockout mice.

\section{References}

1. Hanauer SB: Inflammatory bowel disease: Epidemiology, pathogenesis, and therapeutic opportunities. Inflamm Bowel Dis 12 (Suppl 1): S3-S9, 2006.

2. Xavier RJ and Podolsky DK: Unravelling the pathogenesis of inflammatory bowel disease. Nature 448: 427-434, 2007.

3. Strober W, Fuss I and Mannon P: The fundamental basis of inflammatory bowel disease. J Clin Invest 117: 514-521, 2007.

4. Naito Y, Takagi T and Yoshikawa T: Molecular fingerprints of neutrophil-dependent oxidative stress in inflammatory bowel disease. J Gastroenterol 42: 787-798, 2007.

5. Iborra M, Moret I, Rausell F, et al: Role of oxidative stress and antioxidant enzymes in Crohn's disease. Biochem Soc Trans 39: 1102-1106, 2011.

6. Margoshes M and Vallee BL: Flame photometry and spectrometry; principles and applications. Methods Biochem Anal 3: 353-407, 1956

7. Kojima Y and Hunziker PE: Amino acid analysis of metallothionein. Methods Enzymol 205: 419-421, 1991.

8. Minami T, Yoshita C, Tanaka M, Kubo K, Okabe N and Okazaki Y: Separation of metallothionein isoforms of mouse liver cytosol by capillary zone electrophoresis. Talanta 46: 347-354, 1998. 
9. Coyle P, Philcox JC, Carey LC and Rofe AM: Metallothionein: the multipurpose protein. Cell Mol Life Sci 59: 627-647, 2002.

10. Alvarez L, Gonzalez-Iglesias H, Garcia M, Ghosh S, Sanz-Medel A and Coca-Prados M: The stoichiometric transition from $\mathrm{Zn}_{6} \mathrm{Cu}_{1}$-metallothionein to $\mathrm{Zn}_{7}$-metallothionein underlies the up-regulation of metallothionein (MT) expression: quantitative analysis of MT-metal load in eye cells. J Biol Chem 287: 28456-28469, 2012.

11. Takano H, Inoue $\mathrm{K}$, Yanagisawa $\mathrm{R}$, et al: Protective role of metallothionein in acute lung injury induced by bacterial endotoxin. Thorax 59: 1057-1062, 2004.

12. Ashino T, Arima Y, Shioda S, Iwakura $Y$, Numazawa $S$ and Yoshida T: Effect of interleukin-6 neutralization on CYP3A11 and metallothionein-1/2 expressions in arthritic mouse liver. Eur J Pharmacol 558: 199-207, 2007.

13. Pedersen DS, Fredericia PM, Pedersen MO, et al: Metallic gold slows disease progression, reduces cell death and induces astrogliosis while simultaneously increasing stem cell responses in an EAE rat model of multiple sclerosis. Histochem Cell Biol 138: 787-802, 2012

14. Inoue K, Takano H, Shimada A, et al: Role of metallothionein in coagulatory disturbance and systemic inflammation induced by lipopolysaccharide in mice. FASEB J 20: 533-535, 2006.

15. Takano H, Satoh M, Shimada A, Sagai M, Yoshikawa T and Tohyama C: Cytoprotection by metallothionein against gastroduodenal mucosal injury caused by ethanol in mice. Lab Invest 80 : 371-377, 2000

16. Mita M, Satoh M, Shimada A, et al: Metallothionein is a crucia protective factor against Helicobacter pylori-induced gastric erosive lesions in a mouse model. Am J Physiol Gastrointest Liver Physiol 294: G877-G884, 2008.

17. Kimura T, Itoh N, Takehara M, et al: Sensitivity of metallothionein-null mice to LPS/D-galactosamine-induced lethality. Biochem Biophys Res Commun 280: 358-362, 2001.

18. Klosterhalfen B, Tons C, Hauptmann S, et al: Influence of heat shock protein 70 and metallothionein induction by zinc-bis-(DLhydrogenaspartate) on the release of inflammatory mediators in a porcine model of recurrent endotoxemia. Biochem Pharmacol 52 $1201-1210,1996$

19. Oz HS, Chen T, de Villiers WJ and McClain CJ: Metallothionein overexpression does not protect against inflammatory bowe disease in a murine colitis model. Med Sci Monit 11: BR69-BR73, 2005.

20. Tran CD, Ball JM, Sundar S, Coyle P and Howarth GS: The role of zinc and metallothionein in the dextran sulfate sodiuminduced colitis mouse model. Dig Dis Sci 52: 2113-2121, 2007.

21. Korenaga D, Takesue F, Kido K, et al: Impaired antioxidant defense system of colonic tissue and cancer development in dextran sulfate sodium-induced colitis in mice. J Surg Res 102: 144-149, 2002.

22. Vowinkel T, Kalogeris TJ, Mori M, Krieglstein CF and Granger DN: Impact of dextran sulfate sodium load on the severity of inflammation in experimental colitis. Dig Dis Sci 49: $556-564,2004$

23. Melgar S, Karlsson A and Michaelsson E: Acute colitis induced by dextran sulfate sodium progresses to chronicity in C57BL/6 but not in $\mathrm{BALB} / \mathrm{c}$ mice: correlation between symptoms and inflammation. Am J Physiol Gastrointest Liver Physiol 288 : G1328-G1338, 2005

24. Schepp-Berglind J, Atkinson C, Elvington M, Qiao F, Mannon P and Tomlinson S: Complement-dependent injury and protection in a murine model of acute dextran sulfate sodium-induced colitis. J Immunol 188: 6309-6318, 2012.

25. Michalska AE and Choo KH: Targeting and germ-line transmission of a null mutation at the metallothionein I and II loci in mouse. Proc Natl Acad Sci USA 90: 8088-8092, 1993

26. Takagi T, Naito Y, Uchiyama K, et al: Carbon monoxide liberated from carbon monoxide-releasing molecule exerts an anti-inflammatory effect on dextran sulfate sodium-induced colitis in mice. Dig Dis Sci 56: 1663-1671, 2011.
27. Naito $Y$, Katada $K$, Takagi $T$, et al: Rosuvastatin, a new HMG-CoA reductase inhibitor, reduces the colonic inflammatory response in dextran sulfate sodium-induced colitis in mice. Int J Mol Med 17: 997-1004, 2006.

28. Cooper HS, Murthy SN, Shah RS and Sedergran DJ: Clinicopathologic study of dextran sulfate sodium experimental murine colitis. Lab Invest 69: 238-249, 1993.

29. Howarth GS, Xian CJ and Read LC: Predisposition to colonic dysplasia is unaffected by continuous administration of insulin-like growth factor-I for twenty weeks in a rat model of chronic inflammatory bowel disease. Growth Factors 18: $119-133,2000$

30. Smith P, Mangan NE, Walsh CM, et al: Infection with a helminth parasite prevents experimental colitis via a macrophage-mediated mechanism. J Immunol 178: 4557-4566, 2007.

31. Siegmund B, Lehr HA, Fantuzzi G and Dinarello CA: IL-1 betaconverting enzyme (caspase-1) in intestinal inflammation. Proc Natl Acad Sci USA 98: 13249-13254, 2001.

32. Grisham MB, Hernandez LA and Granger DN: Xanthine oxidase and neutrophil infiltration in intestinal ischemia. Am J Physiol 251: G567-G574, 1986.

33. Ban K and Kozar RA: Protective role of p70S6K in intestinal ischemia/reperfusion injury in mice. PloS One 7: e41584, 2012.

34. Bamba S, Andoh A, Ban H, et al: The severity of dextran sodium sulfate-induced colitis can differ between dextran sodium sulfate preparations of the same molecular weight range. Dig Dis Sci 57: 327-334, 2012.

35. Smith P, Siddharth J, Pearson R, et al: Host genetics and environmental factors regulate ecological succession of the mouse colon tissue-associated microbiota. PloS One 7: e30273, 2012.

36. Mulder TP, Verspaget HW, Janssens AR, de Bruin PA, Pena AS and Lamers CB: Decrease in two intestinal copper/zinc containing proteins with antioxidant function in inflammatory bowel disease. Gut 32: 1146-1150, 1991

37. Kruidenier L, Kuiper I, van Duijn W, et al: Differential mucosal expression of three superoxide dismutase isoforms in inflammatory bowel disease. J Pathol 201: 7-16, 2003.

38. Lih-Brody L, Powell SR, Collier KP, et al: Increased oxidative stress and decreased antioxidant defenses in mucosa of inflammatory bowel disease. Dig Dis Sci 41: 2078-2086, 1996.

39. Bruwer M, Schmid KW, Metz KA, Krieglstein CF, Senninger N and Schurmann G: Increased expression of metallothionein in inflammatory bowel disease. Inflamm Res 50: 289-293, 2001

40. Al-Gindan Y, Shawarby M, Noto A and Taylor CG: Intestinal inflammation in rats induces metallothionein in colonic submucosa. J Clin Biochem Nutr 44: 131-141, 2009.

41. Mehta AJ, Joshi PC, Fan X, et al: Zinc supplementation restores PU.1 and Nrf2 nuclear binding in alveolar macrophages and improves redox balance and bacterial clearance in the lungs of alcohol-fed rats. Alcohol Clin Exp Res 35: 1519-1528, 2011

42. Pankhurst MW, Bennett W, Kirkcaldie MT, West AK and Chung RS: Increased circulating leukocyte numbers and altered macrophage phenotype correlate with the altered immune response to brain injury in metallothionein (MT)-I/II null mutant mice. J Neuroinflammation 8: 172, 2011.

43. Otani K, Watanabe T, Tanigawa T, et al: Anti-inflammatory effects of IL-17A on Helicobacter pylori-induced gastritis. Biochem Biophys Res Commun 382: 252-258, 2009.

44. Yang Z, Ding J, Yang C, et al: Immunomodulatory and antiinflammatory properties of artesunate in experimental colitis. Curr Med Chem 19: 4541-4551, 2012.

45. Kamada N, Hisamatsu T, Honda H, et al: TL1A produced by lamina propria macrophages induces Th1 and Th17 immune responses in cooperation with IL-23 in patients with Crohn's disease. Inflamm Bowel Dis 16: 568-575, 2010. 